\title{
Fine Mapping of the 15q21 Region Implicates TP53BP1 and B2M in the Lymphomagenesis of Follicular and Diffuse Large B-Cell Lymphomas
} Aya-Bonilla Carlos ${ }^{1}$, Camilleri Emily ${ }^{1,2}$, Benton Miles ${ }^{1}$, Haupt Larisa $\mathbf{M}^{1}$, Marlton Paula ${ }^{3}$, Lea Rod $\mathbf{A}^{1}$, Gandhi Maher $\mathbf{K}^{3,4}$ and Griffiths Lyn $\mathbf{R}^{1 *}$

${ }^{1}$ Genomics Research Centre, Institute of Health and Biomedical Innovation, Queensland University of Technology, QId Australia ${ }^{2}$ Department of Orthopedic Surgery, Mayo Clinic, Rochester, MN, USA

${ }^{3}$ Department of Haematology, Princess Alexandra Hospital, Woollongabba, Australia

${ }^{4}$ Centre for Experimental Haematology, Translational Research Institute, Woollongabba, Australia

\begin{abstract}
Background and aim: Recent studies focusing on the discovery of common alterations across cases suffering from follicular lymphoma ( $\mathrm{FL}$ ) and diffuse large B-cell lymphoma (DLBCL) have reported loss of heterozygosity (LOH) and deletions events targeting the $15 q 21$ region, indicating the relevance of this region in the lymphomagenesis of FL and DLBCL. Herein, we investigated the genetic structure of this region by studying identified LOH and copy-loss events and examined this region in the lymphomagenesis of $F L$ and $D L B C L$.
\end{abstract}

Methods: Fine mapping of the genomic region between the 15q15.1 and 15q21.1 loci was performed using data from copy number variation (CNV) and high resolution $L O H$ analyses of $F L(n=21)$ and DLBCL $(n=21)$ cases. Validation of $\mathrm{LOH}$ of this region was performed using microsatellites followed by quantitative-PCR (qPCR) to measure the transcriptional abundance of TP53BP1 and B2M. Also, direct sequencing of exons 1 and 2 of $B 2 M$ was performed on tumor DNA from $24 \mathrm{FL}$ and 23 DLBCL samples.

Results: The integration of $\mathrm{LOH}$ and CNV data identified copy-loss alterations at the $15 \mathrm{q} 21$ loci spanning a 7.5 $\mathrm{Mb}$ region, covering two $\mathrm{LOH}$ regions, termed $\mathrm{LOH}-1$ and $\mathrm{LOH}-2$. The $\mathrm{LOH}-1$ region spans $3.4 \mathrm{Mb}$ and contains 53 genes, from which TP53BP1 (tumor-protein-p53-binding-protein-1) and B2M (Beta-2-Microglobulin) were identified as the most likely target genes due to their roles in DNA double strand break (DSB) repair and immune recognition, respectively. Expression analyses revealed a significant up-regulation of TP53BP1 in NHL with LOH, but no significant changes in B2M expression were observed. Direct sequencing of exons 1 and 2 in $B 2 M$ in FL and DLBCL identified two monoallelic microdeletions associated with DLBCL.

Conclusion: This study identified that deletion mapping to the $15 q 21$ locus cover two LOH regions. LOH of the TP53BP1 and B2M genes appear to be common alterations in FL and DLBCL tumorigenesis.

Keywords: LOH; Copy-loss events; TP53BP1; B2M; DLBCL and FL lymphomagenesis

\section{Introduction}

Follicular lymphoma (FL) and diffuse large B-cell lymphoma (DLBCL) are responsible for over $60 \%$ of newly diagnosed cases of Non-Hodgkin's lymphoma (NHL) [1,2]. FL and DLBCL, have indolent and aggressive clinical courses, respectively; however, they share the same origin (Germinal B-cell centre), but exhibit high heterogeneity in their morphology, clinical outcome and genetic makeup. Approximately $20 \%$ of FL cases transform to DLBCL, characterised by the acquisition of a wide array of secondary alterations, including structural genomic alterations (i.e. translocations including c-MYC, BCL6 genes) and genetic mutations (e.g. TP53), following constitutive BCL-2 overexpression, as a result of the translocation, $t(14 ; 18)$ (q32;q21) [3-6]. Nonetheless, despite differences in genetic aberrations underlying the diverse tumor biology of FL and DLBCL, the presence of common genomic alterations between FL and DLBCL indicates that lymphomagenesis of FL and DLBCL may be caused by inactivation or activation of shared genes and pathways, which can be crucial for survival and development of these B-cell lymphomas [7].

The identification of novel tumor suppressor genes (TSGs) and their role in oncogenesis is paramount for a better understanding of the etiology and pathogenesis of cancer. TSGs negatively regulate vital pathways for the proliferation and sustenance of the malignant phenotype of cancer cells; as such, they are often targets of genomic and genetic alterations that disrupt their function to trigger oncogenic processes. Loss of heterozygosity $(\mathrm{LOH})$ analysis is a useful tool for the identification and study of inactivated TSGs by copy-loss (hemizygous deletions) or copy-neutral events (chromosomal duplications, genomic or epigenetic mutations) [8,9]. The recent use of SNP array data from an unpaired cohort of tumors has enabled the inference of $\mathrm{LOH}$ events in tumor samples without the need for comparison to their normal counterparts $[8,10]$.

Using high resolution $\mathrm{LOH}$ analysis, we recently reported $\mathrm{LOH}$ in common regions across FLs and DLBCLs including candidate tumor suppressor genes (TSGs) within these regions [11]. The precise identification of genes within these commonly affected regions is relevant for a better understanding of the common pathogenic mechanisms that may play a role in the lymphomagenesis of FL and DLBCL. One of the most common LOH regions, in FL and DLBCL

*Corresponding author: Prof. Lyn R. Griffiths, Institute of Health and Biomedical Innovation, Queensland University of Technology, Musk Ave, Kelvin Grove QLD, 4059, Australia, Tel: +617 3138 6102; Fax: +617 3138 6039; E-mail: Iyn.griffiths@qut.edu.au

Received September 25, 2014; Accepted October 27, 2014; Published October 31,2014

Citation: Carlos AB, Emily C, Miles B, Larisa MH, Paula M, et al. (2014) Fine Mapping of the 15q21 Region Implicates TP53BP1 and B2M in the Lymphomagenesis of Follicular and Diffuse Large B-Cell Lymphomas. J Carcinog Mutagen 5: 195. doi:10.4172/2157-2518.1000195

Copyright: @ 2014 Carlos AB, et al. This is an open-access article distributed under the terms of the Creative Commons Attribution License, which permits unrestricted use, distribution, and reproduction in any medium, provided the original author and source are credited. 
cases, spanned the chromosomal bands $15 \mathrm{q} 15.1$ and $15 \mathrm{q} 21$ [11]. $\mathrm{LOH}$ of this genomic region has also been described in pediatric glioblastomas [12]. Several alterations within and surrounding this region has also been described in B-cell lymphomas. In a previous study, integrating copy number and gene expression data analyses, we identified that loss of $15 \mathrm{q} 21$ was a shared alteration between FLs and DLBCLs [7]. Additionally, deletion of both $15 q$ and $8 p$ was correlated with poor prognosis in DLBCL cases, and loss of $15 \mathrm{q} 23$ has been considered as a secondary hit in the transformation of FL to DLBCL $[3,13]$. Furthermore, a previous paired high resolution $\mathrm{LOH}$ study identified a $70 \mathrm{Mb}$ region (15q13.3-15q26.3) as a target of LOH-driving copy-neutral events in FL [14].

Hence, based on the discovery that the deletion of $15 \mathrm{q} 21$ and $\mathrm{LOH}$ of the region $15 \mathrm{q} 15.1-15 \mathrm{q} 21$ are common alterations across FLs and DLBCLs and the potential for TSGs located within this region to play a crucial role in the lymphomagenesis of these B-cell lymphomas $[7,11]$, we undertook copy number and $\mathrm{LOH}$ analyses, integrating this data for fine-mapping of this region. This approach was used to determine overlapping regions affected by $\mathrm{LOH}$ and copy-loss events in these NHL subtypes, and to identify candidate TSGs targeted by these events. By undertaking this integrative approach, we identified that the region targeted by copy-loss events spans $7.5 \mathrm{Mb}$ and contains two specific $\mathrm{LOH}$ regions, named as LOH-1 and LOH-2, which span a region of 3.4 $\mathrm{Mb}$ and $1.1 \mathrm{Mb}$, respectively. We then focused our studies on the LOH- 1 region and identified the TP53BP1 (tumor protein $\mathrm{p} 53$ binding protein 1) and $B 2 M$ (Beta-2-Microglobulin) genes as commonly inactivated TSGs in FLs and DLBCLs. Interestingly, TP53BP1 and B2M have previously been implicated in the lymphomagenesis of DLBCL [15-19]. However, this study supports very recent evidence implicating the inactivation of $B 2 M$ in FL transformation and DLBCL pathogenesis $[15,20,21]$, and it is the first study to suggest a role for TP53BP 1 in the lymphomagenesis of both FL and DLBCL. In addition, two monoallelic microdeletions were identified by sequence screening on exons 1 and 2 of $B 2 M$ in FL and DLBCL, suggesting that somatic mutations are not the common LOH drivers of LOH events targeting this TSG.

\section{Materials and Methods}

\section{Patients and controls}

Genomic DNA and total RNA were isolated from fresh-frozen tumor biopsies of NHL patients and from normal tissue samples of controls, following previously described methodologies $[7,10,11]$. All tumor tissue samples were collected at diagnosis, excluding relapsed/ refractory cases. Cases with transformed FL or FL grade IIIB as well as cases with immunosuppression-associated lymphomas were not included in this study.

\section{Fine mapping of the genomic region 15q15.1-15q21.1}

Previously published data from copy number variation and high resolution LOH analyses of DLBCL $(n=21)$ and FL $(n=21)$ cases was used to perform fine mapping of the chromosomal region between the $15 \mathrm{q} 15.1$ and $15 \mathrm{q} 21.1$ loci to identify genes within this segment targeted by copy-loss (deletions) and LOH events across FLs and DLBCLs $[7,11]$. An integrative analysis of these two genomic approaches was carried out by enrichment of an LOH-Manhattan plot on chromosome 15 with the False Discovery Rates (FDR) Q-values from Genomic Identification of Significant Targets in Cancer (GISTIC) scores [7,11]. Fine-mapping of this region was carried out using the Integrative Genomics Viewer (IGV, v1.5) program.

A statistical overlap approach using a hypergeometric distribution of all genes located within the LOH-1 and LOH-2 regions was performed to investigate and overlap these $\mathrm{LOH}$ genes with collections of curated gene sets, including canonical pathways, Biocarta, KEGG, and Reactome, from the Molecular Signature Database (MsigDB, v3.1). The significance level for this analytical approach was set at $\alpha<0.05$ with a FDR $<0.05$

\section{$T P 53 B P 1$ and $B 2 M$ expression analyses}

TP53BP1 and B2M mean fluorescence intensity values from an Illumina Sentrix Human-6 (v2.0) array of 14 FLs and 17 DLBCLs with available $\mathrm{LOH}$ data were analyzed as previously described [11]. In order to investigate the effect LOH on TP53BP1 and B2M expression, TP53BP1 or B2M expression was compared between cases with RET (retention) and LOH. Cell of origin (COO) subclassification of DLBCL samples was based on criteria established by Shipp [22], resulting in 10 out 21 DLBCL cases classified as GCB-like subtype and 11 out of 21 as non-GCB-like subtype.

Transcript abundance of TP53BP1 and $B 2 M$ was measured by quantitative PCR (qPCR). Briefly, cDNA was reverse transcribed from total RNA isolated from tonsil samples of 6 healthy individuals and from 41 tumor tissues with high resolution $\mathrm{LOH}$ data as previously described [11]. Expression levels of TP53BP1 and B2M were determined by normalization with to RPL13A using the relative quantification $\left(2^{-\Delta \Delta C t}\right)$ method. The oligonucleotide sequences used were: TP53BP1-F (5'-TTGCCATGCCAACCAGCTCCAG-3'), TP53BP1-R (5'-TTCACGGGGTTGCCAGTCCAG-3'), B2M-F (5'-TGCCTGCCGTGTGAACCATGT-3'),B2M-R (5'-TGCGGCATCTTCAAACCTCCATGA-3'), RPL13A-F (5'-ATCTTGTGAGTGGGGCATCT-3') and $R P L 13 A-R$ (5'-CCCTGTGTACAACAGCAAGC-3'). All qPCR reactions were performed as previously described [11]. Expression levels of TP53BP1 and B2M were compared between NHL cases with LOH and RET calls using an independent t-test $(\alpha<0.05)$.

\section{Sequence analysis}

Exons 1 and 2 of $B 2 M$ were sequenced in gDNA from the tumor samples of $24 \mathrm{FL}$ and 23 DLBCL cases. Oligonucleotide sequences used were: Exon 1_F (5'-GGCGCCGATGTACAGACAGCA-3'), exon 1_R (5'-GGCGGGCCACCAAGGAGAAC-3'), exon 2_F (5'-GGAGGTGGCTTGTTGGGAAGGT-3') and exon 2_R (5'-AGATGGGATGGGACTCATTCAGGGT-3'). Bidirectional direct sequencing of $40 \mathrm{ng}$ of the cleaned PCR amplicon was carried out using the BigDye Terminator cycle sequencing kit v3.1 in a GeneScan ${ }^{\circledR} 3130$ (Applied Biosystems, Austin, TX, USA), following the manufacturer's guidelines. Mutational analysis of $B 2 M$ sequences was performed using Geneious Basic software (version 5.5.6, Biomatters).

\section{Results}

Fine mapping of the 15q15.1 - 15q21 genomic region reveals that LOH of TP53BP1 and B2M genes are common alterations in DLBCLs and FLs

LOH data for chromosome 15 was extracted from a previously performed high resolution LOH analysis on SNP array data of NHL cases. $\mathrm{LOH}$ of the region comprising the chromosomal bands $15 \mathrm{q} 15.1$ and $15 \mathrm{q} 21.1$ was identified from this data, as previously reported, and was common across DLBCL and FL cases [11] (Figure 1A). Enrichment of this LOH region with the GISTIC false discovery rates (FDR) for deletion obtained from Green et al., [7], determined that this $\mathrm{LOH}$ region is part of a larger genomic region of $7.5 \mathrm{Mb}$, targeted by common copy-loss alterations across both NHL subtypes (Figure 1B). 
Citation: Carlos AB, Emily C, Miles B, Larisa MH, Paula M, et al. (2014) Fine Mapping of the 15q21 Region Implicates TP53BP1 and B2M in the Lymphomagenesis of Follicular and Diffuse Large B-Cell Lymphomas. J Carcinog Mutagen 5: 195. doi:10.4172/2157-2518.1000195

A
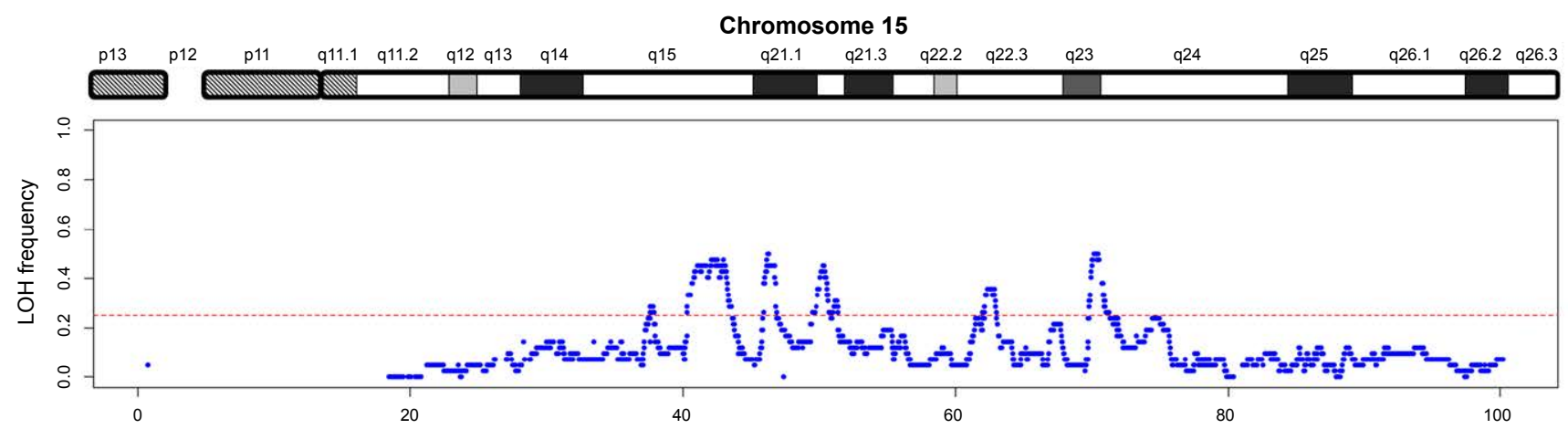

B

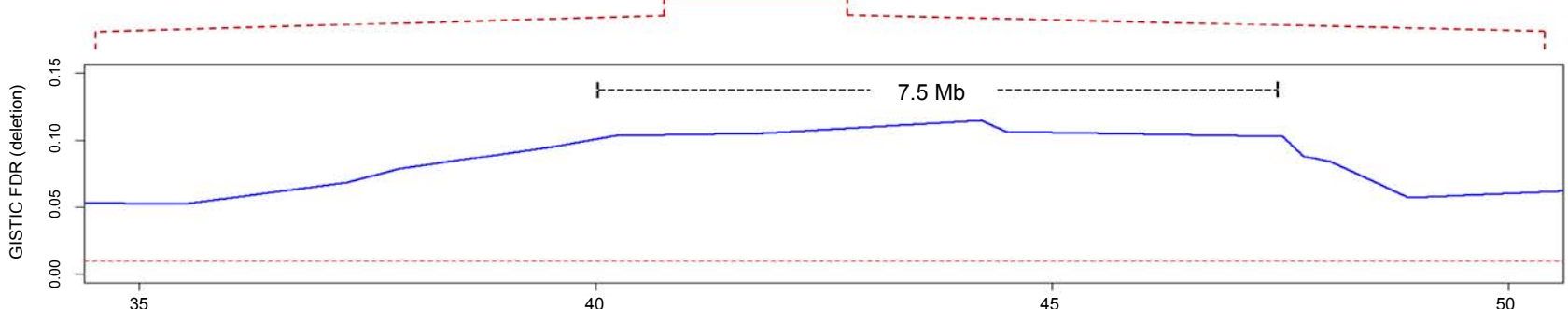

C

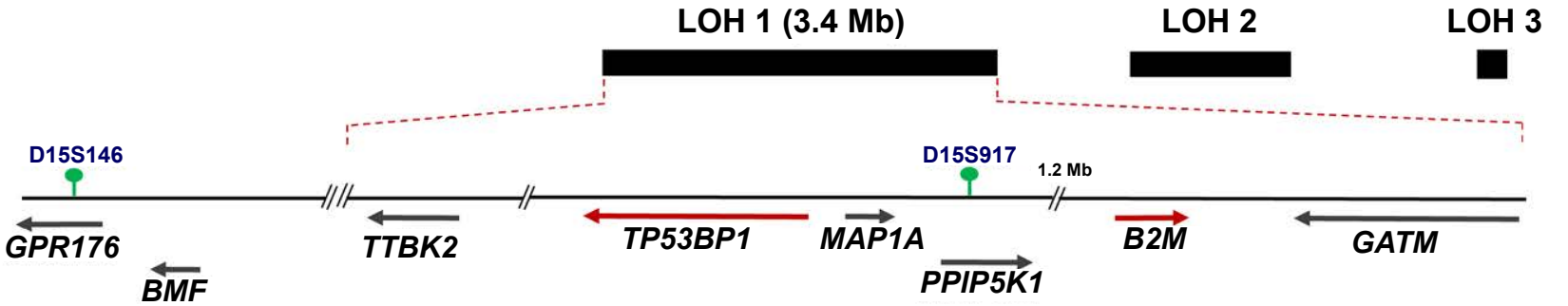

Figure 1: Fine mapping of the chromosomal region 15q15.1-15q21.1 revealed that this region is a common target in FLs and DLBCLs. A) A larger genomic region of $7.5 \mathrm{Mb}$ (hg18.chr15:40190047-47715466) was found to be commonly targeted by copy-loss events across NHL cases, using the False Discovery Rates (FDR) Q-values from GISTIC scores for deletions, previously obtained by Green et al. [7]. B) In addition, it was determined that this commonly deleted region contained 2 LOH regions, LOH-1 region spanning over 3.4 Mb (hg18.chr15:40355785-43764621) and containing 53 genes, and LOH-2 region (hg18.chr15:49759265-50872400) covering a region of $1.1 \mathrm{Mb}$ and harboring 14 genes. Additionally, we observed a third LOH region (LOH-3), which is not further described in this manuscript as it is located outside of the region targeted by copy-loss events. C) Interestingly, the genes TP53BP1 and $\mathrm{B} 2 \mathrm{M}$ were identified to be targeted by LOH events in the LOH-1 region. Localization of microsatellites (D15S146 and D15S917) used for validation of the LOH within the region 15q15.1-15q21.1 are indicated.

Integration of $\mathrm{LOH}$ and $\mathrm{CNV}$ data determined that two $\mathrm{LOH}$ regions, termed LOH-1 and LOH-2, are located within this commonly deleted region (Figure $1 \mathrm{C}$ ), suggesting that $\mathrm{LOH}$ events target more specific, small regions than copy-loss events and that copy-loss events do not necessarily drive $\mathrm{LOH}$.

Fine-mapping determined that the $\mathrm{LOH}-1$ region spans $3.4 \mathrm{Mb}$ and comprises of approximately 53 genes; whereas, the $\mathrm{LOH}-2$ region covers a $1.1 \mathrm{Mb}$ genomic portion that contains 14 genes (Table S1). Statistical overlapping of genes located within both $\mathrm{LOH}$ regions found a significant enrichment of Arginine and Proline metabolism genes (hsa00330; $\mathrm{k} / \mathrm{K}$ score $=0.0556 ; P=0.0000176 ;$ FDR q-value $=0.0255$ ) in LOH-1 including GATM (glycine amidinotransferase), CKMT1A (creatine kinase, mitochondrial 1A) and CKMT1B (creatine kinase, mitochondrial 1B). Loss of heterozygosity of the LOH-1 region was validated by a significant decrease of heterozygosity rates for microsatellites targeting the LOH-1 region in an unmatched cohort of controls and NHL cases (Data not shown).

Amongst the 53 genes located within the LOH-1 region (Table S1), the tumor suppressor genes TP53BP1 (Tumor Protein p53
Binding Protein 1) and B2M (Beta-2-Microglobulin) were identified as the most likely target genes of LOH-driving events in FLs as well as in DLBCLs. This was based on the role that TP53BP1 and B2M play in mechanisms such as DNA double strand break (DSB) repair and immune recognition, and the previously implicated role of these TSGs in the lymphomagenesis of DLBCL [15-19]. In addition, the location of the microsatellite D15S917 (located between the TP53BP1 and B2M loci) validated previously identified LOH associated with these TSGs and implicated this as a common event in the lymphomagenesis of FL and DLBCL. As such, we selected these two genes for more detailed analyses. LOH of TP53BP1 in 45\% (19/42) of total NHL cases, in $43 \%$ $(9 / 21)$ of DLBCL cases and in $48 \%(10 / 21)$ of FL cases. LOH of B2M was also detected in $43 \%(18 / 42)$ of total NHL cases, in $48 \%(10 / 21)$ of DLBCL cases and in 38\% (8/21) of FL cases. In addition, 58\% (11 out of 19 ) and $61 \%$ (11 out of 18 ) of cases exhibited LOH for both TP53BP1 and $B 2 M$, respectively.

\section{Effect of LOH on expression of TP53BP1 and B2M}

TP53BP1 was significantly upregulated in NHL cases with $\mathrm{LOH}$ of TP53BP1: Initial analysis of available TP53BP1 mean fluorescence 
A

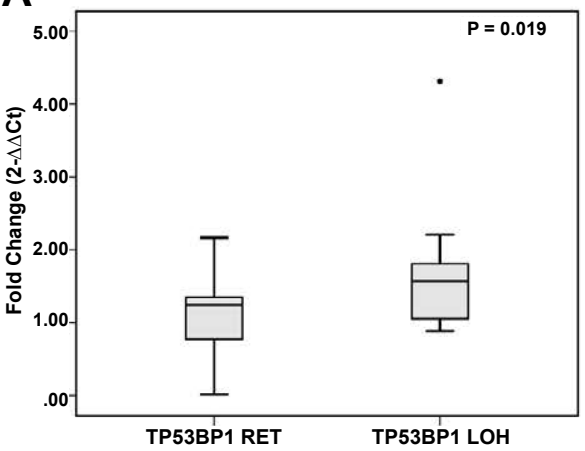

B

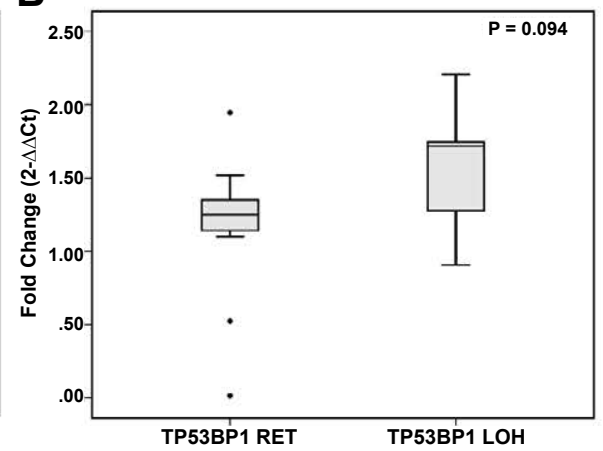

C

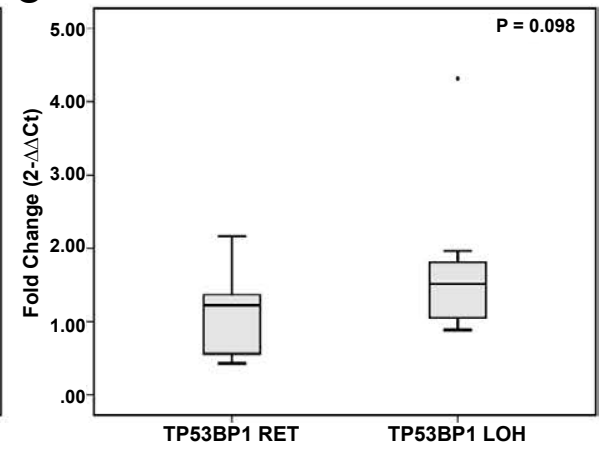

Figure 2: Up-regulation of TP53BP1 was observed in NHL cases with LOH, but not in FLs and DLBCLs; however, a trend was towards upregulation was observed in these NHL subtypes with LOH. Transcript abundance of TP53BP1 was assessed by qPCR in A) NHL ( $n=41), B) F L(n=20)$ and C) DLBCL ( $n=21)$ cases with loss of heterozygosity $(\mathrm{LOH})$ or retention (RET) of TP53BP1.

A

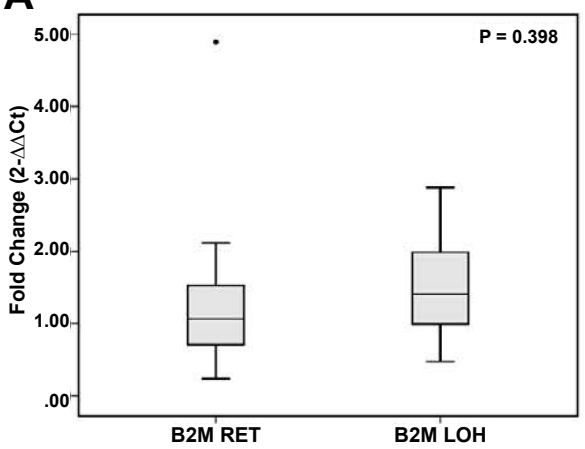

B

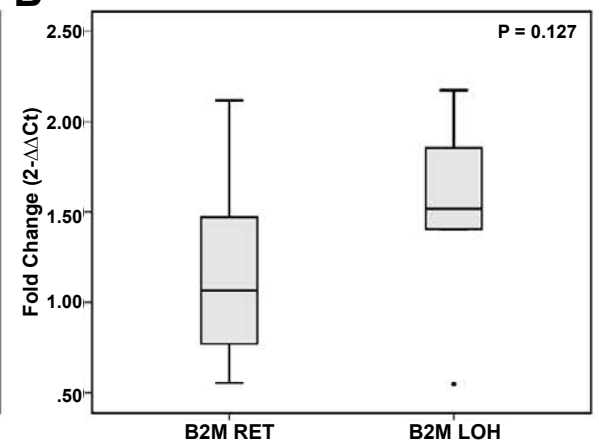

C

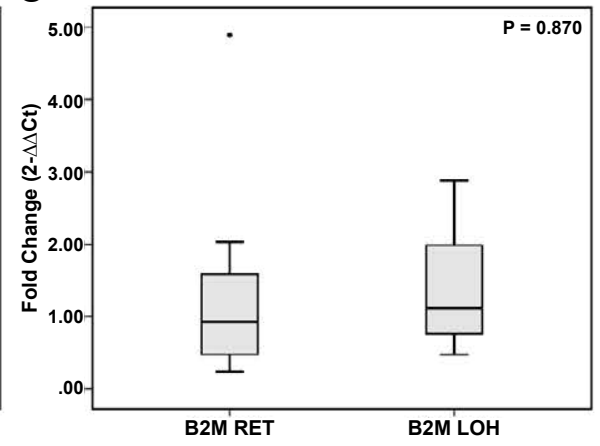

Figure 3: Evaluation of transcript abundance of $B 2 M$ by qPCR in NHL cases with available LOH status data did not show significant differences between cases with RET or LOH in A) NHL $(n=41), B)$ FL $(n=20)$ and C) DLBCL $(n=21)$.

intensity values, from an Illumina Sentrix Human-6 (v2.0) Expression Beadchip, from patients with known LOH status (31 out of $42 \mathrm{NHL}$ cases) [7], did not reveal any significant effect of LOH on TP53BP1 expression between cases with RET and LOH calls in NHLs (847 vs. 872; $P=0.715$ ), FLs ( 795 vs. $882 ; P=0.442$ ) and DLBCL (891 vs. 862 ; $P=0.718$ ) (Figure $\mathrm{S} 1$ ). However, when we examined TP53BP1 gene expression by qPCR in 41 NHL cases with available LOH data, a significant up-regulation of TP53BP1 in NHL cases with $\mathrm{LOH}$ (1.1 vs. 1.6; $P=0.019)$ with trends towards up-regulation in FL (1.15 vs. 1.53; $P=0.094)$ and DLBCL (1.10 vs. $1.71 ; P=0.098)$ cases with LOH (Figure 2 ) was demonstrated. In addition, although not significant, there was a trend toward up-regulation in GCB-like cases $(0.82$ vs. $1.90 ; P=0.157)$ but not in non-GCB-like cases ( 1.20 vs. $1.0 ; P=0.687$ ) (Figure $\mathrm{S} 2$ ).

Loss of heterozygosity did not show a significant effect on B2M expression in FLs and DLBCLs: By comparing B2M mean fluorescence intensity values [7] between NHL cases with RET and LOH, no significant difference was observed in NHL (17661 vs. 17136; $P=0.688$ ), FL ( 16727 vs. $18142 ; P=0.293$ ) or DLBCL ( 18783 vs. 16532 ; $P=0.311$ ) cases (Figure S3). In addition, analysis of $B 2 M$ expression by qPCR showed no significant difference between cases with $\mathrm{LOH}$ and RET calls in NHLs ( 1.23 vs. $1.47 ; P=0.398)$, FLs ( 1.16 vs. $1.54 ; P=0.127$ ) and DLBCLs (1.33 vs. $1.41 ; P=0.870$ ) (Figure 3 ). $\mathrm{LOH}$ events had no significant effect on $B 2 M$ expression in GCB-like DLBCL cases $(0.76$ vs. $1.50 ; P=0.109)$ and non-GCB-like DLBCL cases (1.71 vs. $1.28 ; P=657$ ) (Figure S4).
Monoallelic microdeletions in B2M found in two DLBCL cases with LOH: To determine whether somatic mutations are the driving cause of $\mathrm{LOH}$ associated with $B 2 \mathrm{M}$ in our case cohort, we performed sequence screening in exons 1 and 2 of the B2M gene in 47 NHLs, including $24 \mathrm{FLs}$ and 23 DLBCLs. Exons 1 and 2 of the B2M gene were selected for this mutational screening as a previous report only identified somatic mutations in the exons 1 and 2 but not in the exon 3 of $B 2 M$ [15]. Thus, as a result of this sequence screening performed in our cohort, no mutations were found in FLs; however, a novel mutation $\Delta 12 \mathrm{bp}$ (77-88 bp) with an insT ( $76-77 \mathrm{bp}$ ) was identified in one DLBCL case (Figure 4A). In addition, a $\triangle \mathrm{CT}$ (98-99 bp) deletion was identified in another DLBCL patient (Figure $4 \mathrm{~B}$ ). Both $\Delta 12 \mathrm{bp}$ (77$88 \mathrm{bp}$ ) and $\Delta \mathrm{CT}$ (98-99 bp) are frame-shift mutations, located in exon 1 , and induce premature stop codons producing truncated proteins (51aa and 54aa respectively) with an associated loss of the functional MHC class I domain (28-83 residues).

\section{Discussion}

The integration of genomic data from copy number and high resolution LOH analyses of tumor DNA from FL and DLBCL cases $[7,11]$, allowed us to perform fine mapping of the region between the chromosomal bands 15q15.1 and 15q21.1. Using this approach, we found that the deleted region mapping at $15 \mathrm{q} 21$, previously reported as a common alteration across FLs and DLBCLs [7], spans 7.5 Mb and contains two $\mathrm{LOH}$ regions, termed $\mathrm{LOH}-1$ and $\mathrm{LOH}-2$. These findings 
Citation: Carlos AB, Emily C, Miles B, Larisa MH, Paula M, et al. (2014) Fine Mapping of the 15q21 Region Implicates TP53BP1 and B2M in the Lymphomagenesis of Follicular and Diffuse Large B-Cell Lymphomas. J Carcinog Mutagen 5: 195. doi:10.4172/2157-2518.1000195

suggest that LOH drivers could be more target-specific than copy-loss events [14], possibly by inactivating regions harboring TSGs. Moreover, it also suggests that copy-loss events do not exclusively drive $\mathrm{LOH}$, as these two $\mathrm{LOH}$ regions are separated by a region with the same deletion rate. Recently, the detection of this $7.5 \mathrm{Mb}$ deleted region was further validated by the identification of a deletion-targeted region of $6.2 \mathrm{Mb}$ at the $15 \mathrm{q} 21.1$ locus in a large cohort of DLBCL cases [23], which mostly overlaps with the $7.5 \mathrm{Mb}$ region reported in this study. However, this $6.2 \mathrm{Mb}$ region encompasses the $\mathrm{LOH}-1$ region but excludes the $\mathrm{LOH}-2$ region [23]. The LOH-1 and $\mathrm{LOH}-2$ regions detected in the present study span $3.4 \mathrm{Mb}$ and $1.1 \mathrm{Mb}$, respectively. Furthermore, a significant reduction of heterozygosity rates for a microsatellite in NHL cases validated the $\mathrm{LOH}$ observed in $\mathrm{LOH}-1$. The $\mathrm{LOH}-1$ region contains approximately 53 genes with significant enrichment observed for the Arginine and Proline metabolism pathway. This indicates that this $\mathrm{LOH}$ region may play an important role in the lymphomagenesis of FL and DLBCL, as inactivation of genes within this region can disrupt or reprogram metabolic pathways including the glutamine-prolinearginine circuit, potentially enabling highly efficient malignant B-cell metabolic activity $[24,25]$.

Amongst the genes located within LOH-1, we identified the tumor suppressor genes TP53BP1 and B2M, located at $15 \mathrm{q} 15.3$ and $15 \mathrm{q} 21$ respectively. These TSGs have been previously implicated in DLBCL lymphomagenesis [15-18,23]. However, this is the first study to implicate TP53BP1 and B2M in FL lymphomagenesis where we have identified LOH-driving events targeting these TSGs in DLBCLs and FLs. Furthermore, we observed that although TP53BP1 and B2M are located within a common $\mathrm{LOH}$ region (LOH-1 region) and their $\mathrm{LOH}$ calls are significantly correlated, there is a discrepancy of over $30 \%$ of LOH calls for these two TSGs, suggesting the driving mechanism of LOH may differ between TP53BP1 and B2M. In fact, the higher frequencies of $\mathrm{LOH}$ of TP53BP1 and $B 2 \mathrm{M}$ compared to the previously reported frequencies for the deletion of the 15q21.1 locus in FL and DLBCL cases [7] indicate that LOH of these TSGs are not only driven by copy-loss events. Hence, the genetic mechanisms underlying $\mathrm{LOH}$ of TP53BP1 and B2M in the tumorigenesis of FL and DLBCL as well as the effect of $\mathrm{LOH}$-driving events on the inactivation of these TSGs require further investigation.

The present study implicates $\mathrm{LOH}$ of TP53BP1 and its potential inactivation, as a common event in the lymphomagenesis of both FL and DLBCL. Several lines of evidence indicate that TP53BP1 can play an important role in B-cell lymphomagenesis. For example, singlecopy deletions targeting the TP53BP1 locus in DLBCL cases involve TP53BP1 haploinsufficiency in DLBCL pathogenesis [18]. TP53BP1 participates in the rejoining of double strand breaks (DSBs) induced during normal B-cell development, such as class switch recombination (CSR), V(D)J recombination and non-homologous end-joining (NHEJ) [26-34]. Hence, TP53BP1 inactivation may contribute to the high frequency of translocations involving immunoglobulin heavy chain loci with oncogenes (i.e. BCL2, BCL6 and MYC) observed in B-cell lymphomas [35-38]. In murine models, haploinsufficiency of TP53BP1 induced genomic instability and impaired DNA double strand break (DSB) repair, has been demonstrated to correlate with a high incidence of thymic and B-cell lymphomas [29,31]. The implication of LOH of TP53BP1 in FL lymphomagenesis suggests a crucial role for TP53BP1 $\mathrm{LOH}$ as the secondary hit in the transformation of FL to DLBCL, driving the acquisition of secondary genomic and genetic alterations through disruption of DSB repair mechanisms in CSR during B-cell development $[3,4,32,36]$. However, it is likely that $\mathrm{LOH}$ of TP53BP1 has a synergistic effect with TP53 silencing during FL transformation, resulting in the reduced response to DSB of FL cells undergoing malignant transformation [5,39]. This is supported by the observed significant increase in tumor development, genomic instability and lymphomagenesis in mice lacking TP53 expression resulting in null or reduced TP53BP1 expression [29,33]. In addition, recent evidence showing that copy-number alterations (CNAs) perturb the TP53 pathway in DLBCLs [23], suggest that LOH of TP53BP1 may also play a role in disruption of the TP53 pathway, influencing FL transformation as well as DLBCL pathogenesis. Moreover, TP53 and TP53BP1 might also be implicated in DLBCL chemoresponsiveness as cases with deletions of 8p, 15q (TP53BP1) and 17p (TP53) exhibit poor responses to R-CHOP-21 therapy [13].

A significant up-regulation of TP53BP1 was observed in NHL cases with $\mathrm{LOH}$. This is in contrast to the significant down-regulation of TP53BP1 in DLBCL cases with hemizygous deletions of the TP53BP1 locus [18], supporting the hypothesis that $\mathrm{LOH}$ of TP53BP1 may be the result of copy-neutral events in FL and in DLBCL. However, although we observed TP53BP1 up-regulation in our LOH case cohort, the concept "LOH" corresponds to the functional inactivation of a TSG by genetic
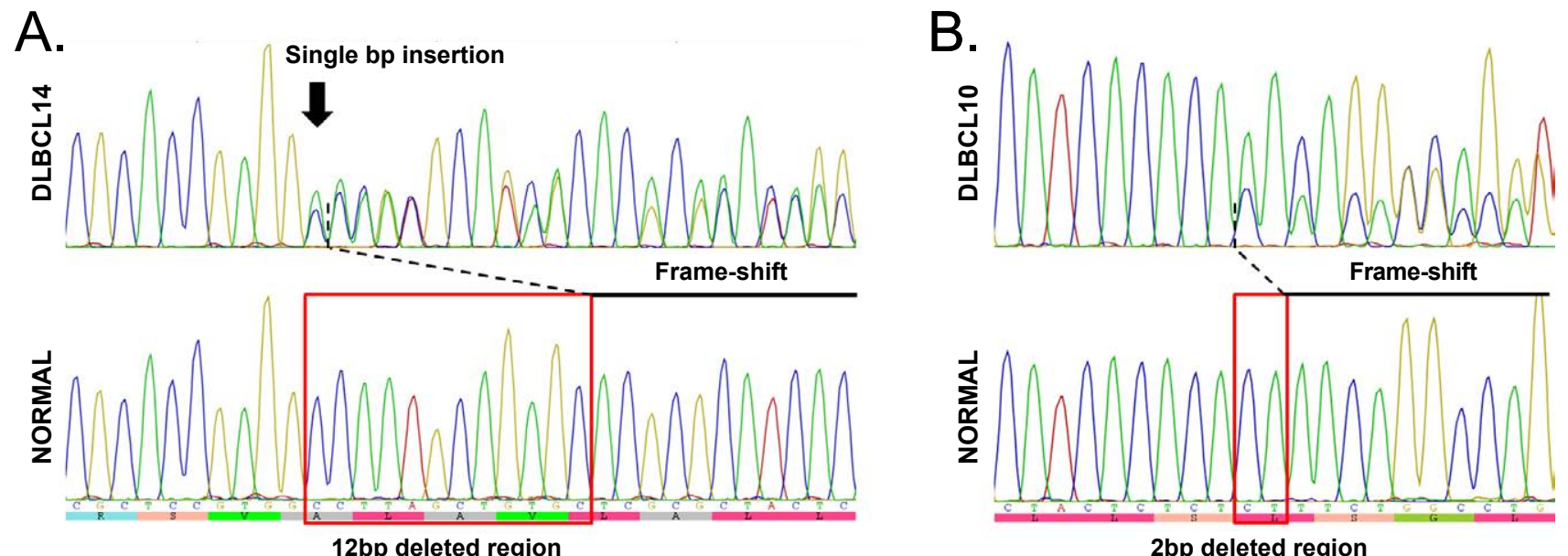

Figure 4: Mutational screening of exons 1 and 2 of $B 2 M$ across 24 FLs and 23 DLBCLs identified A) a novel monoallelic $\Delta 12 \mathrm{bp}$ (77-88 bp) with an insT (76-77 bp) in a DLBCL patient and B) a $\triangle$ CT (98-99 bp) in another DLBCL case. 
mechanisms (copy-neutral or copy-loss events) in a broader cellular perspective, rather than a down-regulatory effect (happloinsufficiency) $[9,40]$. Thus, up-regulation of TP53BP1 may be due to the cellular response against $\mathrm{LOH}$-driving events causing inactivation of TP53BP1, or aberrant transcriptional mechanisms induced by copy-neutral events (duplication, somatic mutations). Therefore, additional "driver" alterations of TP53BP1 LOH events as well as the effect of these on the TP53BP1 function, need to be further examined as somatic mutations targeting the TP53BP1 locus not identified in NHL cases by previous whole exome sequencing studies [16,17].

Another TSG identified and studied within this LOH region was the beta-2-microglobulin (B2M) gene. $\mathrm{LOH}$ of $B 2 M$ herein identified as a common event in FL and DLBCL has been reported in colorectal and bladder carcinomas, as well as in testicular and central nervous system (CNS) DLBCL $[41,42]$. Recent studies have reported that B2M is widely targeted and inactivated by somatic mutations and deletions in DLBCL cases $[15-17,41,42]$. Additionally, recent lines of evidence have identified mutations targeting the $B 2 M$ locus in a small fraction of transformed FL cases $[20,21]$. In the present study, mutational screening of exons 1 and 2 of B2M in FL and DLBCL cases, detected two monoallelic microdeletions in two DLBCL cases with LOH, one novel $\Delta 12$ bp (77$88 \mathrm{bp}$ ) with an ins $\mathrm{T}(76-77 \mathrm{bp})$ and another previously reported $\triangle \mathrm{CT}$ (98-99 bp). Both deletions are frameshift mutations and predicted to produce two truncated proteins (51 aa and 54 aa respectively) with a loss of the MHC class I domain in both cases. Therefore, it is likely that these mutations inactivate the function of B2M as instability of the truncated B2M proteins would likely have a dominant negative effect on the assembly of the major histocompatibility complex class I (MHC I) complex $[15,43]$. In contrast, based on the detection of only two mutations in two DLBCL patients with $\mathrm{LOH}$ and the lower frequency of deletions in comparison to the frequency of $\mathrm{LOH}$ in our cohort of FLs and DLBCLs, we suggest that additional genetic mechanisms could induce LOH of this TSG, and subsequently drive its inactivation in FLs and DLBCLs. This observation is supported by the low frequency of mutations targeting $B 2 M$ in FL cases undergoing transformation $[20,21]$. In addition, it is important to mention that despite only detecting somatic mutations in 2 out of 23 DLBCL cases, the frequency of these mutations in our population $(8.7 \%)$ is not significantly lower to the observed frequencies of somatic mutations targeting the $B 2 M$ loci in previous studies analyzing a larger cohort of DLBCL cases, which vary between $9 \%$ and $13 \%[15,17]$. Thus, the low frequency of somatic mutations across these studies, including the present study, support our hypothesis suggesting that $\mathrm{LOH}$ events in addition to mutations may also be driving inactivation of this TSG.

The inactivation of $\mathrm{B} 2 \mathrm{M}$, a small protein required for the assembly of the HLA class I molecule, is one of the mechanisms that disables the recognition of the HLA class I complex by CD8+ cytotoxic T-cell lymphocytes (CTL), enabling tumor cells to escape immune surveillance and growth control [15-17,41, 43,44]. Although inactivation of $B 2 M$ has been previously detected in DLBCLs [15-17,41], the demonstration of $\mathrm{LOH}$ of the B2M locus as a common event across FL and DLBCLs suggests that evasion of CTL-mediated regulatory mechanisms may play a role in FL and DLBCL lymphomagenesis. Additionally, using pathway analyses, we have recently reported the direct interaction of B2M with GRB2 (growth factor receptor-bound protein 2), an adaptor protein that activates the RAS-MAPK pathway, and a direct interaction of GRB2 with PTPRJ (protein tyrosine phosphatase receptor type J), a negative regulator of B-cell survival pathways, such as MAPK, PI3K,
VEGF [45-49]. Thus, the presence of LOH events targeting the TSGs $B 2 M$ and $P T P R J$ suggests that $B 2 M$ inactivation is not an isolated event in lymphomagenesis but part of an orchestrated network of oncogenic hits that inactivate key TSGs responsible for the regulation of critical oncogenic pathways and thus, induce the malignant phenotype of these lymphomas [45]. In addition, in this study, the transcript abundance of $B 2 M$ did not differ significantly between cases with retention and $\mathrm{LOH}$. This pattern was also observed, in a previous study, between DLBCL cases with and without inactivation of $B 2 M$, where even the presence of detectable mRNA levels of B2M in cases harboring B2M missense variants expressed significantly low protein levels of B2M [15]. Thus, the no correlation between transcript and protein levels of B2M suggest that despite transcript levels detectable in patients with inactivated copies of $\mathrm{B} 2 \mathrm{M}$, the protein instability resulting from the presence of missense mutations can induce undetectable levels of protein in these cases, as previously suggested [15]. Hence, based on this finding, it is likely that DLBCL cases with $\mathrm{LOH}$ of $B 2 M$ exhibit low protein levels regardless their $B 2 M$ transcript abundance; however, further functional studies will provide a much clearer insight of the effect of LOH in B2M protein levels, in the context of NHL pathogenesis.

In conclusion, the $\mathrm{LOH}$ and potential inactivation of the TSGs located within the 15q15.1-15q21 genomic region may be an important target in the tumorigenesis of FL and DLBCL due to their involvement in key pathways of tumor survival. In addition, this study implicates LOH of TP53BP1 and B2M, identified as a shared alteration across $\mathrm{FL}$ and DLBCL, in the lymphomagenesis and progression of these B-cell lymphomas. However, further genetic and functional studies are required to determine the driving causes of $\mathrm{LOH}$ of TP53BP1 and B2M.

\section{Statement of Author Contributions}

$\mathrm{CAB}$ designed experiments, performed experiments, analyzed data and drafted the manuscript. EC performed experiments and analyzed data. MB analyzed data and drafted the manuscript. LMH and PM designed experiments, whilst RL analyzed data. MKG designed experiments and analyzed data. LRG coordinated the research and designed experiments, analyzed data and wrote the manuscript.

\section{Acknowledgments}

We thank all participating institutions and patients for supporting our research. This study was supported by funding from the Cancer Council of Queensland, the Genomics Lymphoma Project and the Herbert family. The Australasian Leukaemia and Lymphoma Group (ALLG) Tissue Bank is supported by the Leukaemia Foundation of Australia and the National Health and Medical Research Council.

\section{References}

1. Jayasekara $\mathrm{H}$, Karahalios A Juneja S, Thursfield V, Farrugia $\mathrm{H}$, et al. (2010) Incidence and survival of lymphohematopoietic neoplasms according to the World Health Organization classification: a population-based study from the Victorian Cancer Registry in Australia. Leuk Lymphoma 51: 456-468.

2. Chisté M, Vrotsos E, Zamora C, Martinez A (2013) Chronic lymphocytic leukemia/small lymphocytic lymphoma involving the aortic valve. Ann Diagn Pathol 17: 295-297.

3. Martinez-Climent JA, Alizadeh AA, Segraves R, Blesa D, Rubio-Moscardo F, et al. (2003) Transformation of follicular lymphoma to diffuse large cell lymphoma is associated with a heterogeneous set of DNA copy number and gene expression alterations. Blood 101: 3109-3117.

4. Carlotti E, Wrench D, Matthews J, Iqbal S, Davies A, et al. (2009) Transformation of follicular lymphoma to diffuse large B-cell lymphoma may occur by divergent evolution from a common progenitor cell or by direct evolution from the follicular lymphoma clone. Blood 113: 3553-3557.

5. Lo Coco F, Gaidano G, Louie DC, Offit K, Chaganti RS, et al. (1993) p53 mutations are associated with histologic transformation of follicular lymphoma. Blood 82: 2289-2295. 
Citation: Carlos AB, Emily C, Miles B, Larisa MH, Paula M, et al. (2014) Fine Mapping of the 15q21 Region Implicates TP53BP1 and B2M in the Lymphomagenesis of Follicular and Diffuse Large B-Cell Lymphomas. J Carcinog Mutagen 5: 195. doi:10.4172/2157-2518.1000195

Page 7 of 8

6. Bende RJ, Smit LA, van Noesel CJ (2007) Molecular pathways in follicular lymphoma. Leukemia 21: 18-29.

7. Green MR, Aya-Bonilla C, Gandhi MK, Lea RA, Wellwood J, et al. (2011) Integrative genomic profiling reveals conserved genetic mechanisms for tumorigenesis in common entities of non-Hodgkin's lymphoma. Genes Chromosomes Cancer 50: 313-326.

8. Beroukhim R, Lin M, Park Y, Hao K, Zhao X, et al. (2006) Inferring loss-ofheterozygosity from unpaired tumors using high-density oligonucleotide SNP arrays. PLoS Comput Biol 2: e41.

9. Tischfield JA (1997) Loss of heterozygosity or: how I learned to stop worrying and love mitotic recombination. Am J Hum Genet 61: 995-999.

10. Green MR, Jardine P, Wood P, Wellwood J, Lea RA, et al. (2010) A new method to detect loss of heterozygosity using cohort heterozygosity comparisons. BMC Cancer 10: 195.

11. Aya-Bonilla C, Green MR, Camilleri E, Benton M, Keane C, et al. (2013) Highresolution loss of heterozygosity screening implicates PTPRJ as a potential tumor suppressor gene that affects susceptibility to Non-Hodgkin's lymphoma. Genes Chromosomes Cancer 52: 467-479.

12. Qu HQ, Jacob K, Fatet S, Ge B, Barnett D, et al. (2010) Genome-wide profiling using single-nucleotide polymorphism arrays identifies novel chromosomal imbalances in pediatric glioblastomas. Neuro Oncol 12: 153-163.

13. Scandurra M, Mian M, Greiner TC, Rancoita PM, De Campos CP, et al. (2010) Genomic lesions associated with a different clinical outcome in diffuse large B-Cell lymphoma treated with R-CHOP-21. Br J Haematol 151: 221-231.

14. Cheung KJJ, Delaney A, Ben-Neriah S, Schein J, Lee T, et al. (2010) High resolution analysis of follicular lymphoma genomes reveals somatic recurrent sites of copy-neutral loss of heterozygosity and copy number alterations that target single genes. Genes, Chromosomes and Cancer 49: 669-681.

15. Challa-Malladi M, Lieu YK, Califano O, Holmes AB, Bhagat G, et al. (2011) Combined genetic inactivation of $\beta 2-$ Microglobulin and CD58 reveals frequent escape from immune recognition in diffuse large B cell lymphoma. Cancer Cell 20: $728-740$

16. Morin RD, Mendez-Lago M, Mungall AJ, Goya R, Mungall KL, et al. (2011) Frequent mutation of histone-modifying genes in non-Hodgkin lymphoma. Nature 476: 298-303.

17. Pasqualucci L, Trifonov V, Fabbri G, Ma J, Rossi D, et al. (2011) Analysis of the coding genome of diffuse large B-cell lymphoma. Nat Genet 43: 830-837.

18. Takeyama K, Monti S, Manis JP, Dal Cin P, Getz G, et al. (2008) Integrative analysis reveals 53BP1 copy loss and decreased expression in a subset of human diffuse large B-cell lymphomas. Oncogene 27: 318-322.

19. Lohr JG, Stojanov P, Lawrence MS, Auclair D, Chapuy B, et al. (2012) Discovery and prioritization of somatic mutations in diffuse large B-cel lymphoma (DLBCL) by whole-exome sequencing. Proc Natl Acad Sci U S A 109: 3879-3884

20. Okosun J, Bödör C, Wang J, Araf S, Yang CY, et al. (2014) Integrated genomic analysis identifies recurrent mutations and evolution patterns driving the initiation and progression of follicular lymphoma. Nat Genet 46: 176-181.

21. Pasqualucci $L$, Khiabanian $H$, Fangazio $M$, Vasishtha $M$, Messina $M$, et al. (2014) Genetics of follicular lymphoma transformation. Cell Rep 6: 130-140.

22. Shipp MA, Ross KN, Tamayo P, Weng AP, Kutok JL, et al. (2002) Diffuse large B-cell lymphoma outcome prediction by gene-expression profiling and supervised machine learning. Nat Med 8: 68-74.

23. Monti S, Chapuy B, Takeyama K, Rodig SJ, Hao Y, et al. (2012) Integrative analysis reveals an outcome-associated and targetable pattern of p53 and cell cycle deregulation in diffuse large B cell lymphoma. Cancer Cell 22: 359-372.

24. Hanahan D, Weinberg RA (2011) Hallmarks of cancer: the next generation Cell 144: 646-674.

25. Phang JM, Liu W, Hancock C, Christian KJ (2012) The proline regulatory axis and cancer. Front Oncol 2: 60.

26. Arakawa H, Hauschild J, Buerstedde JM (2002) Requirement of the activationinduced deaminase (AID) gene for immunoglobulin gene conversion. Science 295: 1301-1306.

27. Manis JP, Morales JC, Xia Z, Kutok JL, Alt FW, et al. (2004) 53BP1 links DNA damage-response pathways to immunoglobulin heavy chain class-switch recombination. Nat Immunol 5: 481-487.

28. Reina-San-Martin B, Chen HT, Nussenzweig A, Nussenzweig MC (2004) ATM is required for efficient recombination between immunoglobulin switch regions. $J$ Exp Med 200: 1103-1110.

29. Ward IM, Difilippantonio S, Minn K, Mueller MD, Molina JR, et al. (2005) 53BP1 cooperates with p53 and functions as a haploinsufficient tumor suppressor in mice. Mol Cell Biol 25: 10079-10086.

30. Ward IM, Minn K, Jorda KG, Chen J (2003) Accumulation of checkpoint protein 53BP1 at DNA breaks involves its binding to phosphorylated histone H2AX. J Biol Chem 278: 19579-19582.

31. Ward IM, Minn K, van Deursen J, Chen J (2003) p53 Binding protein 53BP1 is required for DNA damage responses and tumor suppression in mice. Mol Cell Biol 23: 2556-2563.

32. Ward IM, Reina-San-Martin B, Olaru A, Minn K, Tamada K, et al. (2004) 53BP1 is required for class switch recombination. J Cell Biol 165: 459-464

33. Morales JC, Franco S, Murphy MM, Bassing CH, Mills KD, et al. (2006) 53BP1 and $\mathrm{p} 53$ synergize to suppress genomic instability and lymphomagenesis. Proc Natl Acad Sci U S A 103: 3310-3315.

34. Difilippantonio S, Gapud E, Wong N, Huang CY, Mahowald G, et al. (2008) 53BP1 facilitates long-range DNA end-joining during $V(D) J$ recombination. Nature 456: 529-533.

35. Lenz G, Nagel I, Siebert R, Roschke AV, Sanger W, et al. (2007) Aberrant immunoglobulin class switch recombination and switch translocations in activated B cell-like diffuse large B cell lymphoma. J Exp Med 204: 633-643.

36. Kotani A, Kakazu N, Tsuruyama T, Okazaki IM, Muramatsu M, et al. (2007) Activation-induced cytidine deaminase (AID) promotes B cell lymphomagenesis in Emu-cmyc transgenic mice. Proc Natl Acad Sci U S A 104: 1616-1620.

37. Ci W, Polo JM, Cerchietti L, Shaknovich R, Wang L, et al. (2009) The BCL6 transcriptional program features repression of multiple oncogenes in primary $B$ cells and is deregulated in DLBCL. Blood 113: 5536-5548.

38. Raghavan SC, Swanson PC, Wu X, Hsieh CL, Lieber MR (2004) A non-B-DNA structure at the $\mathrm{Bcl}-2$ major breakpoint region is cleaved by the RAG complex. Nature 428: 88-93.

39. Phan RT, Dalla-Favera R (2004) The BCL6 proto-oncogene suppresses p53 expression in germinal-centre B cells. Nature 432: 635-639.

40. Knudson AG (2001) Two genetic hits (more or less) to cancer. Nat Rev Cancer 1: 157-162.

41. Jordanova ES, Riemersma SA, Philippo K, Schuuring E, Kluin PM (2003) Beta2-microglobulin aberrations in diffuse large B-cell lymphoma of the testis and the central nervous system. Int J Cancer 103: 393-398.

42. Maleno I, Aptsiauri N, Cabrera T, Gallego A, Paschen A, et al. (2011) Frequent loss of heterozygosity in the î2-microglobulin region of chromosome 15 in primary human tumors. Immunogenetics 63: 65-71.

43. Hill DM, Kasliwal T, Schwarz E, Hebert AM, Chen T, et al. (2003) A dominant negative mutant beta 2-microglobulin blocks the extracellular folding of a major histocompatibility complex class I heavy chain. J Biol Chem 278: 5630-5638.

44. Jordanova ES, Riemersma SA, Philippo K, Giphart-Gassler M, Schuuring $E$, et al. (2002) Hemizygous deletions in the HLA region account for loss of heterozygosity in the majority of diffuse large B-cell lymphomas of the testis and the central nervous system. Genes, Chromosomes and Cancer 35: 38-48.

45. Aya-Bonilla C, Camilleri E, Haupt LM, Lea R, Gandhi MK, et al. (2014) In silico analyses reveal common cellular pathways affected by loss of heterozygosity $(\mathrm{LOH})$ events in the lymphomagenesis of Non-Hodgkin's lymphoma (NHL). BMC Genomics 15: 390.

46. Grazia Lampugnani M, Zanetti A, Corada M, Takahashi T, Balconi G, et al (2003) Contact inhibition of VEGF-induced proliferation requires vascular endothelial cadherin, beta-catenin, and the phosphatase DEP-1/CD148. J Cell Biol 161: 793-804

47. Sacco F, Tinti M, Palma A, Ferrari E, Nardozza AP, et al. (2009) Tumor suppressor density-enhanced phosphatase-1 (DEP-1) inhibits the RAS pathway by direct dephosphorylation of ERK1/2 kinases. J Biol Chem 284 22048-22058. 
Citation: Carlos AB, Emily C, Miles B, Larisa MH, Paula M, et al. (2014) Fine Mapping of the 15q21 Region Implicates TP53BP1 and B2M in the Lymphomagenesis of Follicular and Diffuse Large B-Cell Lymphomas. J Carcinog Mutagen 5: 195. doi:10.4172/2157-2518.1000195

Page 8 of 8

48. Wang J, Yuan Y, Zhou Y, Guo L, Zhang L, et al. (2008) Protein interaction data set highlighted with human Ras-MAPK/PI3K signaling pathways. J Proteome Res 7: 3879-3889.
49. Tsuboi N, Utsunomiya T, Roberts RL, Ito H, Takahashi K, et al. (2008) The tyrosine phosphatase CD148 interacts with the p85 regulatory subunit of phosphoinositide 3-kinase. Biochem J 413: 193-200. 